OPEN ACCESS

Edited by:

Jianxun Ding,

Changchun Institute of Applied

Chemistry (CAS), China

Reviewed by:

Quanyin $\mathrm{Hu}$,

Massachusetts Institute

of Technology, United States

Veronica Lazar,

University of Bucharest, Romania

*Correspondence:

Hongliang Xin

xhl@njmu.edu.cn

†These authors have contributed equally to this work

Specialty section: This article was submitted to Experimental Pharmacology and Drug Discovery,

a section of the journal

Frontiers in Pharmacology

Received: 29 October 2018

Accepted: 21 December 2018

Published: 22 January 2019

Citation:

Wang B, Wu W, Lu H, Wang Z and Xin $H$ (2019) Enhanced

Anti-tumor of Pep-1 Modified Superparamagnetic Iron Oxide/PTX

Loaded Polymer Nanoparticles.

Front. Pharmacol. 9:1556

doi: 10.3389/fphar.2018.01556

\section{Enhanced Anti-tumor of Pep-1 Modified Superparamagnetic Iron Oxide/PTX Loaded Polymer Nanoparticles}

\author{
Baoyan Wang ${ }^{1 \dagger}$, Weijun Wu ${ }^{1 \dagger}$, Hongjin $\mathrm{Lu}^{2}$, Zhi Wang ${ }^{3}$ and Hongliang Xin ${ }^{2 *}$ \\ ${ }^{1}$ Nanjing Drum Tower Hospital, The Affiliated Hospital of Nanjing University Medical School, Nanjing, China, ${ }^{2}$ Department \\ of Pharmaceutics, School of Pharmacy, Nanjing Medical University, Nanjing, China, ${ }^{3}$ Department of Pharmacy, The Second \\ Affiliated Hospital of Nanjing Medical University, Nanjing, China
}

Superparamagnetic iron-oxide nanoparticle (SPION) has gained tremendous attention for drug delivery applications due to their unique properties. In this study, we developed a dual targeted delivery system with paclitaxel (PTX) and SPION co-loaded PLGA nanoparticles, modified with Pep-1 peptide (Pep-NP-SPION/PTX), to achieve magnetic targeting and active targeting for tumor treatment. SPION was synthesized by a coprecipitation method and was then encapsulated with PTX simultaneously into PLGA nanoparticles. After that, the non-complex was conjugated with Pep-1 through chemical modification. The resulting Pep-NP-SPION/PTX showed a spherical morphology and an average size of $100 \mathrm{~nm}$. The enhancement cellular uptake of Pep-NP-SPION was demonstrated in in vitro through cell experiments. The $I_{50}$ value of Pep-NP-SPION/PTX and NP-SPION/PTX was determined to be 10.2 and $19.4 \mu \mathrm{g} / \mathrm{mL}$, respectively. A biodistribution study showed that obvious higher accumulations of Pep-NP-SPION was observed in tumors, compared with that of non-targeting nanocomposites. Moreover, under the condition of a magnetic field, both NP-SPION and Pep-NPSPION exhibited much higher tumor distribution. Furthermore, Pep-NP-SPION/PTX presented desirable in vivo anti-tumor effects based on active targeting and magnetic targeting characteristics. Altogether, Pep-NP-SPION/PTX can offer magnetic targeting and receptor mediated targeting to enhance the anti-tumor outcome.

Keywords: tumor, drug delivery system, superparamagnetic iron oxide nanoparticle, interleukin-13 receptor $\alpha 2$, dual targeting nanocarrier

\section{INTRODUCTION}

Current tumor chemotherapy still faces a major problem with the of lack of selectivity of drugs on tumor cells, which leads to a narrow therapeutic index of most anti-tumor drugs (Wong et al., 2016). As a result, the achievement of an adequate therapeutic effect requires a high concentration of antitumor drugs, which in turn enhances the systematic toxicity. Thus, in order to reduce the toxicity of a chemotherapy drug at its minimum dose, specific nanoparticulate drug delivery systems, such as nanoparticle, liposome and polymeric micelle, could provide a non-invasive treatment strategy due to its passive targeting properties (Huang et al., 2016; Muralidharan et al., 2017; 
Roberts et al., 2017). However, these conventional drug delivery systems lack the capability of active targeting for tumor section.

Recently, superparamagnetic iron-oxide nanoparticles (SPION) consisting of cores made of iron oxides, have been considered to be attractive in cancer theranostic applications, since they can be delivered to the required tissue through an external magnetic field (Hachani et al., 2016). Due to the superparamagnetism, a high saturation field and a high field irreversibility, SPION has been widely used for various biological applications ( $\mathrm{Li}$ et al., 2016). Moreover, SPIONs could lose their magnetization and become highly dispersed even after the removal of the magnetic field (Nagesh et al., 2016). However, biological application of SPION was limited because of the high surface hydrophobicity, making them susceptible to being ingested and eliminated by mononuclear phagocyte systems (MPS). In order to prolong the circulation time, it is necessary to modify the surface of SPION by amphiphilic copolymer coating to convert hydrophobic SPION into hydrophilic ones (Mirsadeghi et al., 2016; Silva et al., 2016).

Under an external magnetic field, SPION-encapsulated polymer nanoparticles could easily reach around the tumor section. In order to increase the active targeting of nanoparticles and uptake by tumor cells, receptor-mediated endocytosis could serve as a versatile targeting strategy via linking nanoparticles with multifunctional ligands to construct a potentially multiple targeting drug nanocarrier (Zhao et al., 2015; Rabiej et al., 2016; Shen et al., 2018). It is reported that various receptors are over-expressed on tumor cells, including folate receptor, neuropilin-1 receptor and transferrin receptor (Wang et al., 2011; Du et al., 2015; Song et al., 2015). Based on these receptors, drug delivery systems have been modified with corresponding targeting ligands and explored to deliver drugs through receptormediated endocytosis. The interleukin 13 receptor $\alpha 2$ (IL-13R $\alpha 2$ ) is a subtype of the interleukin-13 receptor family, which is overexpressed on tumor cells (Mintz et al., 2002; Balyasnikova et al., 2012). It has been reported that IL-13R $\alpha 2$, acting as a decoy receptor, has an intimate relationship with the progression of a tumor and can undergo internalization after binding to ligands (Kawakami et al., 2001). This property indicates that IL-13R $\alpha 2$ could serve as a promising targeted moiety for anti-tumor drug delivery.

Pep-1 peptide (CGEMGWVRC) that was screened by the phage display library, could bind to IL-13R $\alpha 2$ with high affinity and specificity and could be exploited to target ligand to tumor cells (Pandya et al., 2012). In our previous study, we demonstrated that Pep-1 conjugated paclitaxel (PTX) loaded nanoparticles, could be internalized into tumor cells via IL$13 \mathrm{R} \alpha 2$ mediated endocytosis (Wang et al., 2014). However, the accumulation of the targeted drug delivery system in the tumor tissue was still rather low and could only be by enhanced penetration and retention (EPR) effects (Wilhelm et al., 2016). Therefore, we aimed at developing PTX and SPION co-loaded polymer nanoparticles with Pep-1 peptide modification as a dual targeting nanocarrier (designated as Pep-NP-SPION/PTX) for tumor treatment in this study. SPION was prepared using a co-precipitation method and loaded into PEG-PLGA polymer nanoparticles that were modified with Pep-1 peptide to form
Pep-NP-SPION/PTX (Figure 1A). As showed in Figure 1B, after intravenous (i.v.) injection, Pep-NP-SPION/PTX was expected to accumulate at the tumor tissue in the presence of an external magnetic field and then be internalized into tumor cells through IL-13R $\alpha 2$ mediated endocytosis, which would reduce the uptake of Pep-NP-SPION/PTX by the MPS and enhance the anti-tumor efficiency of PTX. These physical-chemical properties of the dual targeted nanocarrier were also systematically characterized. Furthermore, the in vitro biological targeted capability of PepNP-SPION/PTX was investigated. Finally, the in vitro and in vivo anti-tumor effect of Pep-NP-SPION/PTX was studied using a cell and subcutaneous xenograft tumor mice model, respectively.

\section{MATERIALS AND METHODS}

\section{Materials}

Qleic acid, Iron(II) chloride, iron(III) chloride and ammonium hydroxide were purchased from Sinopharm Chemical Reagent Co., Ltd. (China). Methoxyl poly(ethylene glycol)-co-poly(D,Llactic-co-glycolic acid) copolymer(MePEG-PLGA, $40 \mathrm{KDa}$ ) and Maleimidyl-poly(ethylene glycol)-co-poly(D,L-lactic- coglycolic acid) copolymer(Male-PEG-PLGA, 41.5 KDa) were purchased from Daigang Biomaterial Co., Ltd. (Jinan, China). PTX was purchased from Zelang Medical Technology Co., Ltd. (Nanjing, China). 3-(4, 5-dimethylthiazol-2-yl)-2, 5-diphenyltetrazolium bromide (MTT) was purchased from Beyotime Biotechnology Co., Ltd. (Nantong, China). Penicillin-streptomycin, RPMI 1640 medium, fetal bovine serum (FBS) and $0.25 \%(\mathrm{w} / \mathrm{v})$ trypsin solution were obtained from Gibco BRL (Gaithersburg, MD, United States).

\section{Cell Line}

The C6 cell line was obtained from the Institute of Biochemistry and Cell Biology, Shanghai Institutes for Biological Sciences, Chinese Academy of Sciences (Shanghai, China). The cell line was cultured in a RPMI 1640 medium, supplemented with 10\% FBS, $1 \%$ penicillin and $100 \mathrm{mg} / \mathrm{mL}$ streptomycin sulfate. Cells were cultured in incubators maintained at $37^{\circ} \mathrm{C}$ with $5 \% \mathrm{CO}_{2}$. All experiments were performed in the logarithmic phase of cell growth.

\section{Animals}

Mice (male, 4-5 weeks, 20-25 g) were supplied by the Department of Experimental Animals, Nanjing Medical University (Nanjing, China) and maintained under standard a housing environment. All animal experiments were performed in accordance with protocols and evaluated and approved by the ethics committee of Nanjing Medical University.

\section{Synthesis of SPION Coated With Oleic Acid (OA-SPION)}

Superparamagnetic iron-oxide nanoparticle was prepared by coprecipitating ferrous and ferric salts in an alkaline medium (Schleich et al., 2013). Briefly, $0.10 \mathrm{~g}$ of $\mathrm{FeCl}_{2} \cdot 4 \mathrm{H}_{2} \mathrm{O}$ and $0.27 \mathrm{~g}$ of $\mathrm{FeCl}_{3} \cdot 6 \mathrm{H}_{2} \mathrm{O}$ were added into $50 \mathrm{~mL}$ PEG600, which was placed 
A
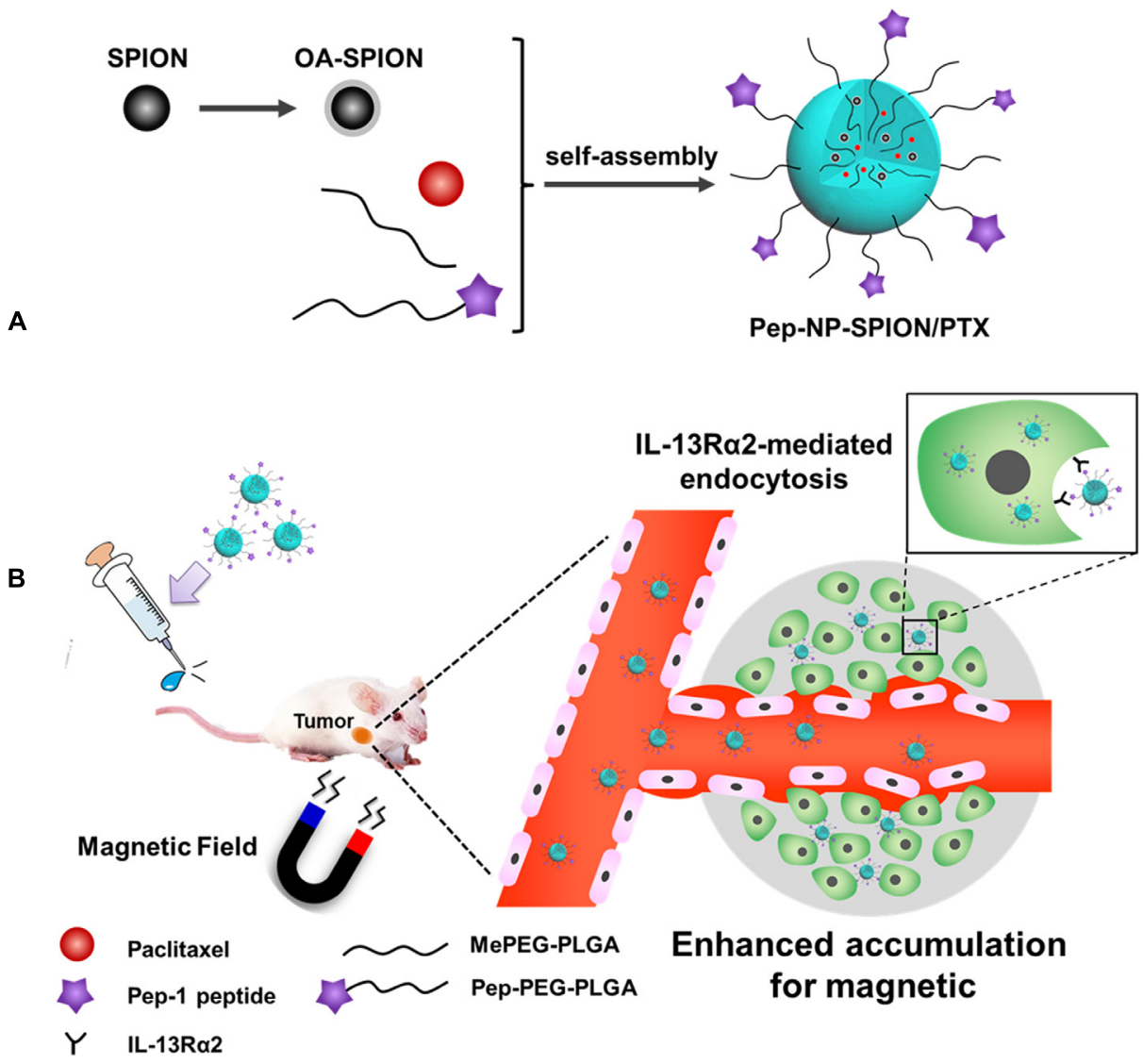

FIGURE 1 | Schematic design of Pep-NP-SPION/PTX. The main components and preparation of the Pep-NP-SPION/PTX (A). After intravenous injection, Pep-NP-SPION/PTX targets to tumor tissue via external magnetic field and then is internalized into tumor cells through IL-13R $\alpha 2$ mediated endocytosis (B).

under a vacuum overnight at $40^{\circ} \mathrm{C}$ to degas. The mixture was then heated to $125^{\circ} \mathrm{C}$ under nitrogen protection. Then, $2 \mathrm{~mL}$ ammonium hydroxide was added, and the mixture was reheated to $125^{\circ} \mathrm{C}$. $5 \mathrm{~min}$ later, $20 \mathrm{~mL}$ of oleic acid was added into the solution at $125^{\circ} \mathrm{C}$ and the mixture was kept at this temperature for another $30 \mathrm{~min}$. Finally, the black precipitate was separated by an external magnet and washed three times with absolute ethanol and ultrapure water to remove the unreacted reagent, respectively. Dry SPION coated with oleic acid was prepared through freeze-drying for further use.

\section{Preparation of Pep-NP-SPION/PTX}

Paclitaxel and SPION loaded PLGA-based nanoparticles were prepared via an emulsion/solvent evaporation method (Wilhelm et al., 2016). Firstly, the conjugation of Pep-1 peptide to PEGPLGA copolymer via the reaction of maleimide with thiol group was carried out as previously reported (Wilhelm et al., 2016). Then, 19 mg MePEG-PLGA and 2 mg Pep-PEG-PLGA polymer were dissolved in $1 \mathrm{~mL}$ dichloromethane (DCM) containing PTX ( $1 \mathrm{mg} / \mathrm{mL}$ ) and OA-SPION (Fe concentration: $4 \mathrm{mg} / \mathrm{mL}$ ). Then the mixture was added to a $2 \mathrm{~mL} 1 \%$ (w/v) sodium cholate aqueous solution, which was finally sonicated using a probe sonicator (Xin Zhi Biotechnology Co., Ltd., China) for $5 \mathrm{~min}$ at $190 \mathrm{~W}$ output. The suspension was added drop-wisely into $10 \mathrm{~mL} 1 \%(\mathrm{w} / \mathrm{v})$ sodium cholate solution under moderate stirring. A rotary evaporator was used to evaporate the redundant DCM at $40^{\circ} \mathrm{C}$. The solutions were centrifuged at $13500 \mathrm{~g}$ for $40 \mathrm{~min}$ to remove the excess excipient. After being washed three times by water, the Pep-NP-SPION/PTX was collected and re-dispersed in PBS for further use.

\section{Characterization of Pep-NP-SPION/PTX Morphology, Particle Size and Zeta Potential of Pep-NP-SPION/PTX}

The morphology of Pep-NP-SPION/PTX was characterized by the transmission electron microscope (TEM, Philips CM 100 ) operating at a voltage of $100 \mathrm{kV}$. The particle size and zeta potential of nanoparticles were assessed by dynamic light scattering (DLS, Zs90, Malvern, United Kingdom).

\section{Determination of SPION Loading Content}

The Fe content was measured using the phenanthroline spectrophotometric method. Lyophilized nanoparticles were dissolved in DCM, and then dried under nitrogen. The residue was dissolved with $10 \%(\mathrm{v} / \mathrm{v}) \mathrm{HCl}$ solution. The yellow solution was combined with aqueous solutions of hydroxylamine 
hydrochloride $(10 \%, \mathrm{w} / \mathrm{v})$ and sodium acetate buffer solution $(\mathrm{pH}=5,1 \mathrm{~mol} / \mathrm{L})$. Then $0.1 \%$ phenanthroline solution was added. After $0.5 \mathrm{~h}$, the absorbance at $510 \mathrm{~nm}$ was measured and the Fe content was determined based on a comparison of a standard curve.

\section{Encapsulation Efficiency and Loading Capacity of PTX}

The amount of PTX was measured by high performance liquid chromatography (HPLC) with UV detection at $227 \mathrm{~nm}$ (LC10AT, SHIMADZU, Japan). The mobile phase consisted of acetonitrile and water $(47: 53, \mathrm{v} / \mathrm{v})$ with a gradient elution pumped at a flow rate of $1.0 \mathrm{~mL} / \mathrm{min}$ and the column was maintained at $30^{\circ} \mathrm{C}$. The calibration curve was linear in the range of $0.1-$ $100 \mu \mathrm{g} / \mathrm{mL}$ with a correlation coefficient of $R^{2}=0.9998$.

To determine the encapsulation efficiency (EE\%) and loading capacity (LC\%) of Pep-NP-SPION/PTX, the lyophilized samples were dissolved in DCM, and then dried under nitrogen. The residue was dissolved in mobile phase solution and the concentration of PTX was analyzed by HPLC as described. The EE\% and LC\% were calculated as indicated below $(n=3)$.

$$
\begin{aligned}
& \mathrm{EE} \%=\frac{\text { Amount of PTX in the nanoparticles }}{\text { Total amount of PTX added }} \times 100 \% \\
& \mathrm{LC} \%=\frac{\text { Amount of PTX in the nanoparticles }}{\text { Nanoparticles weight }} \times 100 \%
\end{aligned}
$$

\section{In vitro Release}

The in vitro release kinetics of PTX from Pep-NP-SPION/PTX was measured by a standard dialysis method in PBS $(0.04 \mathrm{M}, \mathrm{pH}$
7.4) or HAc-NaAc buffer (0.04 M, pH 5.0) containing $0.1 \%(\mathrm{w} / \mathrm{v})$ Tween- 80 at $37^{\circ} \mathrm{C}$. Briefly, nanoparticles were suspended in $1 \mathrm{~mL}$ of mediator solution and placed in a dialysis bag (MWCO 3000). The dialysis bag was immersed in $30 \mathrm{~mL}$ of the release medium which was shaken at $37^{\circ} \mathrm{C}$. At predetermined time intervals, a portion of $0.3 \mathrm{~mL}$ dialysate was harvested, and the same volume of fresh mediator solution was added. The PTX concentration of samples was determined by HPLC as described above.

\section{In vitro Evaluation}

\section{In vitro Cellular Uptake}

C6 cells were seeded into a 24-well plate at the density of $1 \times 10^{5}$ cells per well. After $24 \mathrm{~h}$ incubation, the cells were incubated with NP-SPION and Pep-NP-SPION at different concentrations (Fe concentration: 25 and $50 \mu \mathrm{g} / \mathrm{mL}$ ) at $37^{\circ} \mathrm{C}$ for $1 \mathrm{~h}$, respectively. After that, the cells were washed three times with cold PBS and fixed with $4 \%$ formaldehyde for $10 \mathrm{~min}$. Then, these cells were stained with Pearls' reagent $(2 \%$ potassium ferrocyanide $/ 6 \% \mathrm{HCl}$ : 1/1) for $30 \mathrm{~min}$. Finally, after washing by PBS for three times, the uptake of nanoparticles in the C6 cells was observed using a microscope.

\section{In vitro Cytotoxicity}

An MTT assay was used to evaluate the cell cytotoxicity of Pep-NP-SPION/PTX. C6 cells were seeded into 96-well plates at the density of 5000 cells/well and incubated at $37^{\circ} \mathrm{C}$ in a $5 \% \mathrm{CO}_{2}$ atmosphere, and then incubated with $\mathrm{Taxol}^{\circledR}$, NP-SPION/PTX and Pep-NP-SPION/PTX at different PTX concentration $(1,5,10$, and $20 \mu \mathrm{g} / \mathrm{mL})$ after $24 \mathrm{~h}$, respectively. All the concentrations of Fe used were based on the corresponding PTX concentrations (Fe concentrations: 3.9, 19.6, 39.2, and

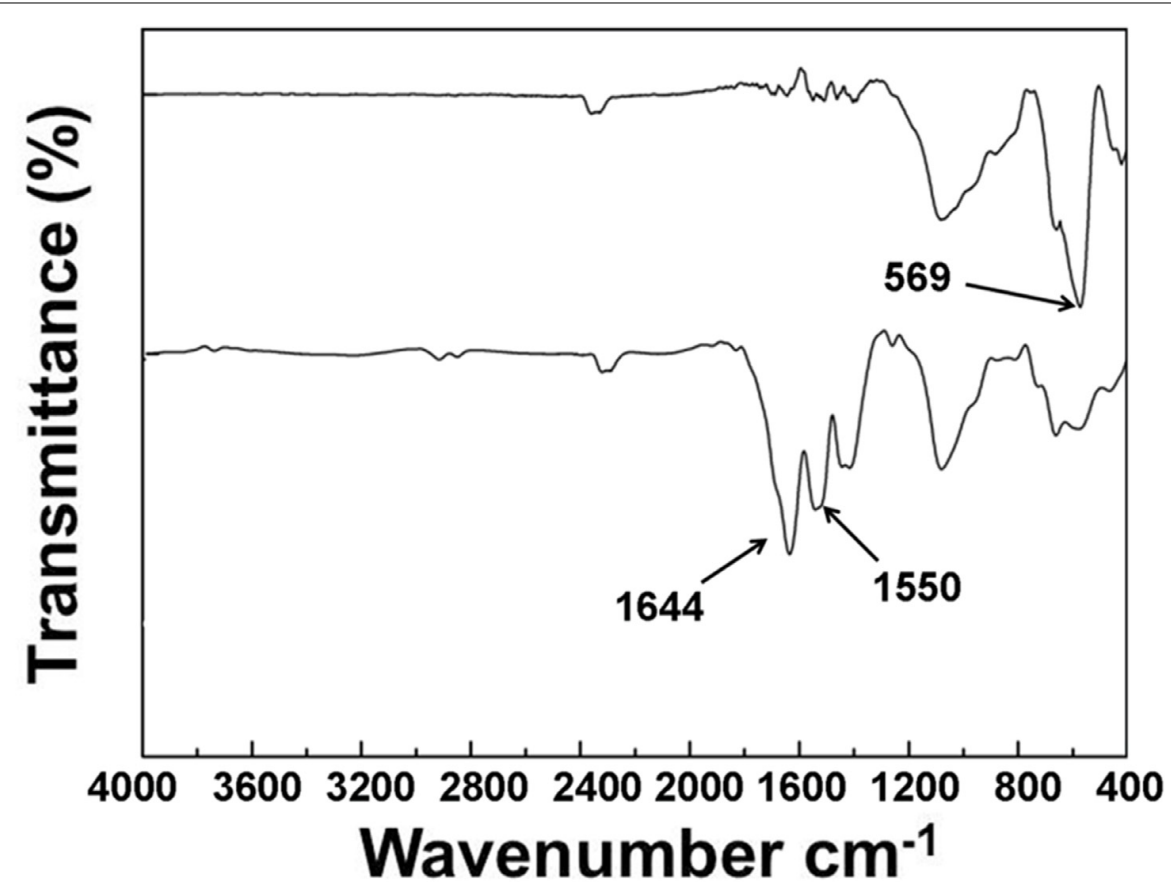

FIGURE 2 | FTIR spectra of SPION and OA-SPION. 
$78.4 \mu \mathrm{g} / \mathrm{mL}$ ). After $48 \mathrm{~h}$, the culture medium was discarded and $20 \mu \mathrm{L}$ MTT solution was added into each well and incubated for additional $4 \mathrm{~h}$. Then the unreacted dye was removed and $200 \mu \mathrm{L}$ of DMSO was added to each well. Finally, the optical density was measured by a microplate reader at wavelength of $490 \mathrm{~nm}$.

\section{In vivo Evaluation \\ Biodistribution of Pep-NP-SPION/PTX in Tumor \\ Tissue}

C6 cells $\left(3 \times 10^{6}\right.$ cells suspended in $100 \mu \mathrm{L}$ PBS $)$ were injected into the armpit of the right anterior limbs of mice subcutaneously. When the tumor volume reached about $300 \mathrm{~cm}^{3}$, the mice were divided into four groups randomly, then intravenously administrated with NP-SPION/PTX and Pep-NPSPION/PTX (Fe dose of $15 \mathrm{mg} / \mathrm{kg}$ ), respectively. The influence of the external magnetic field was also evaluated. $2 \mathrm{~h}$ after injection, the mice were sacrificed, and the tumor tissues were harvested and fixed in $4 \%$ paraformaldehyde. After paraffin embedding, the tumors were cut into $5 \mu \mathrm{m}$ and stained with Prussian blue staining.

\section{In vivo Anti-tumor Efficacy}

In vivo tumor growth inhibition was carried out to evaluate the anti-tumor efficacy of Pep-NP-SPION/PTX. Subcutaneous xenograft tumor mice model was established as described above. With the tumor volume reached about $100 \mathrm{~mm}^{3}$, the mice were divided into six groups randomly, and then intravenously administrated with saline, Taxol ${ }^{\circledR}$, NP-SPION/PTX and PepNP-SPION/PTX (PTX dose: $4.5 \mathrm{mg} / \mathrm{kg}$, Fe dose: $15.6 \mathrm{mg} / \mathrm{kg}$ ), respectively. The influence of external magnetic field was also evaluated. The formulation was given every other day for four injections. 15 days later, the mice were sacrificed with tumor collection and tumor volume was calculated (the formula: $\pi / 6 \times$ larger diameter $\times$ smaller diameter ${ }^{2}$ ).

\section{RESULTS}

\section{Synthesis of SPION Coated With Oleic Acid}

In order to improve the hydrophobicity and dispersibility of SPION, their surfaces were conjugated by oleic acid through physical adsorption. The FTIR spectra of SPION and OA-SPION are shown in Figure 2. The absorption peak at $569 \mathrm{~cm}^{-1}$ was the characteristic absorption peak of Fe-O bond. The spectrum of OA-SPION showed a sharp absorption peak at $1644 \mathrm{~cm}^{-1}$, which was attributed to the $\mathrm{C}=\mathrm{O}$ stretching vibrational absorption of oleic acid. However, the characteristic vibrational absorptions were not present in the spectrum of SPION. These results
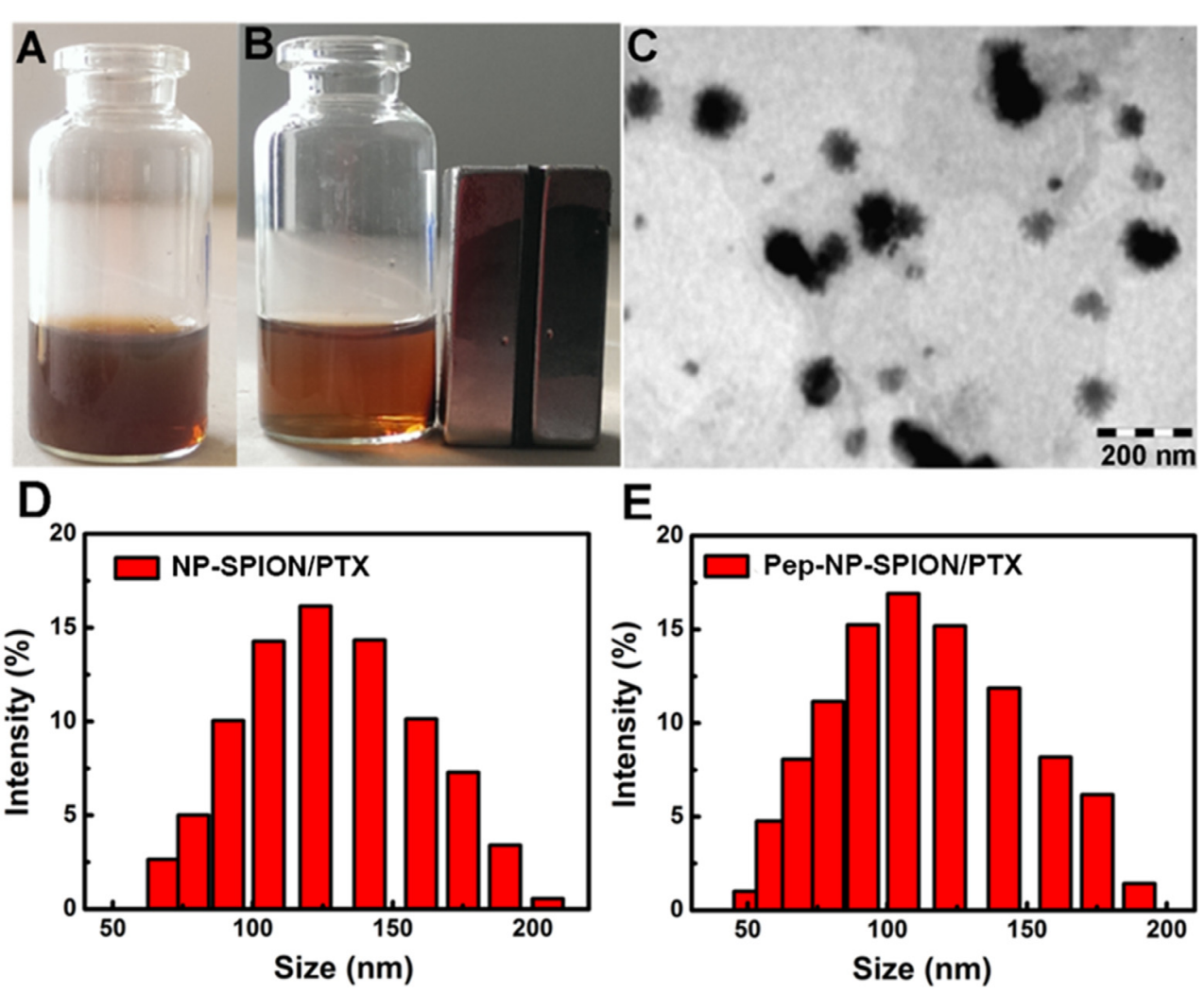

FIGURE 3 | Characterization of Pep-NP-SPION/PTX. Pep-NP-SPION/PTX solution (A) or absorption under external magnetic field (B); TEM image of Pep-NP-SPION/PTX (C) and size distribution of NP-SPION/PTX (D) and Pep-NP-SPION/PTX (E). 
demonstrated the successful modification of oleic acid on the surface of SPION.

\section{Characterization of Pep-NP-SPION/PTX}

Pep-NP-SPION/PTX was prepared via an emulsion/solvent evaporation method. As shown in Figure 3, Pep-NP-SPION/PTX was well-dispersed in water and showed a brown color before magnetic separation. When exposed to a magnetic field, the solution become more and more colorless and the nanoparticles were collected near the magnet. Therefore, the magnetic property of SPION was not affected by the encapsulation of PEGPLGA.

The morphology and size of Pep-NP-SIPION/PTX was characterized by TEM and DLS, respectively. A TEM photograph showed that Pep-NP-SPION/PTX was uniformly spherical and of regular shape with a narrow size distribution. As shown in Table 1, the particle size of NP-SPION/PTX and PepNP-SPION/PTX were $129.8 \pm 1.2 \mathrm{~nm}$ and $101.6 \pm 1.0 \mathrm{~nm}$, respectively. The particle size difference between NPSPION/PTX and Pep-NP-SPION/PTX might be due to the modification of hydrophilic Pep-1 peptides, which enhanced the hydrophilicity of polymer nanoparticles. The zeta potential of NP-SPION/PTX and Pep-NP-SPION/PTX were below $-20 \mathrm{mV}$.

The PTX LC\% and EE\% of Pep-NP-SPION/PTX were $3.54 \pm 0.14 \%$ and $81.30 \pm 3.50 \%$, respectively, compared with $3.22 \pm 0.86 \%$ and $66.85 \pm 1.84 \%$ for NP-SPION/PTX. The change of encapsulation efficiency and loading capacity might be attributed to the modification of polymer materials with Pep-1 peptide. The iron loading of NP-SPION/PTX and Pep-NPSPION/PTX was $12.41 \pm 2.75 \mathrm{mg}$ and $13.53 \pm 3.38 \mathrm{mg} / 100 \mathrm{mg}$ polymer, respectively.

\section{In vitro Drug Release}

The release profiles of PTX-loaded magnetic nanoparticles under different $\mathrm{pH}$ conditions are presented in Figure 4. Tween-80 was used to increase the solubility of PTX in a buffer solution and to avoid the binding of PTX to the nanoparticle surface. Herein, the drug release behavior was studied under a simulated physiological environment $(\mathrm{pH}$ 7.4) and an acidic condition $(\mathrm{pH}$ 5.0) because of the lower $\mathrm{pH}$ value in tumor tissues, due to excess lactic acid produced by hypoxia and acidic intracellular organelles (Xu et al., 2012).

Paclitaxel-loaded magnetic nanoparticles all exhibited an initial burst release profile with total PTX releases of about $60 \%$ before $12 \mathrm{~h}$. After $72 \mathrm{~h}$, NP-SPION/PTX and Pep-NPSPION/PTX showed a total drug release of 63.4 and $65.8 \%$ of PTX at $\mathrm{pH} 7.4$, respectively, while 82.3 and $87.1 \%$ of the PTX in PBS

TABLE 1 | Characterization of NP-SPION/PTX and Pep-NP-SPION/PTX.

\begin{tabular}{lcc}
\hline & NP-SPION/PTX & Pep-NP-SPION/PTX \\
\hline Particle size (nm) & $129.8 \pm 1.2$ & $101.6 \pm 1.0$ \\
Zeta potential (mV) & $-21.9 \pm 0.5$ & $-21.2 \pm 0.5$ \\
Encapsulation efficiency (EE\%) & $66.85 \pm 1.84$ & $81.30 \pm 3.50$ \\
Loading capacity (LC\%) & $3.22 \pm 0.86$ & $3.54 \pm 0.14$ \\
Iron loading (mg/100 mg polymer) & $12.41 \pm 2.75$ & $13.53 \pm 3.38$
\end{tabular}

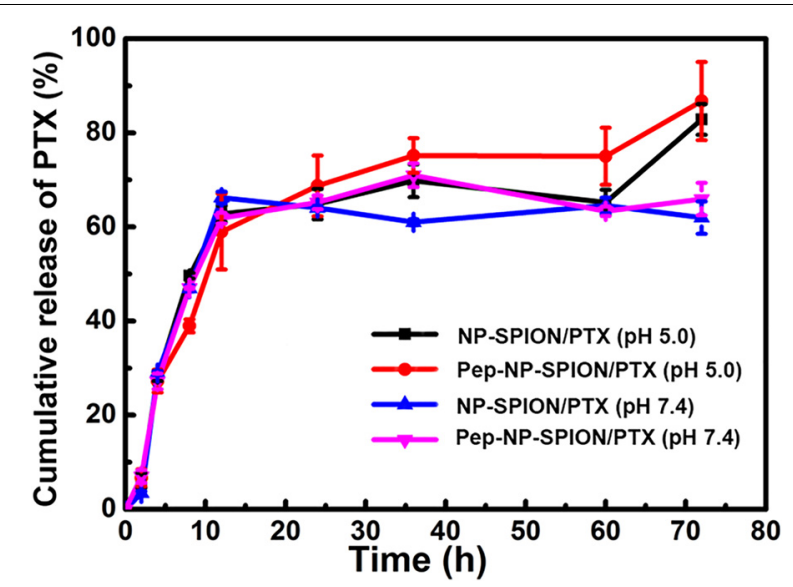

FIGURE 4 | PTX release profiles from NP-SPION/PTX and Pep-NPSPION/PTX in HAc-NaAc (pH 5.0) and PBS (pH 7.4) buffer solution containing $0.1 \%(w / v)$ Tween-80.

( $\mathrm{pH}$ 5.0), respectively. These results suggested that solution $\mathrm{pH}$ could affect the release pattern of PTX from nanoparticles. The $\mathrm{pH}$ responsive release of the dual targeted nanoparticles may be beneficial for enhancing the anti-tumor efficacy of the PTX in the acidic tumor microenvironment.

\section{In vitro Cell Experiment In vitro Cellular Uptake}

It has been reported that IL-13R $\alpha 2$ was upregulated in C6 cell line (Daines et al., 2006), so the C6 cell was used as the cell model to investigate the in vitro uptake of Pep-NPSPION in this study. The cellular uptake of Pep-NP-SPION was studied quantitatively by Prussian blue staining. The number of blue granules within C6 cells was related to the nanoparticle concentration. As shown in Figure 5, the blue granules intensity was increased with the increase of iron concentrations ranging from 25 to $50 \mu \mathrm{g} / \mathrm{mL}$ after $1 \mathrm{~h}$ incubation, which indicated that the cellular uptake of Pep-NP-SPION exhibited a concentrationdependent mode. Moreover, the cellular uptake of Pep-NPSPION was obviously higher than that of NP-SPION. These results implied that the modification of Pep-1 peptide could enhance the cellular uptake of nanoparticles through receptormediated endocytosis.

\section{In vitro Cytotoxicity}

The in vitro cell viability of NP-SPION/PTX and Pep-NPSPION/PTX was evaluated by MTT assay. The cytotoxicity of various PTX formulations exhibited a concentration-dependent pattern with the increase of PTX concentration ranging from 1 to $20 \mu \mathrm{g} / \mathrm{mL}$. Different degrees of cytotoxicity were found in all the PTX formulations. As shown in Figure 6, the cell viability of Pep-NP-SPION/PTX was even lower than $50 \%$ at PTX concentration of $10 \mu \mathrm{g} / \mathrm{ml}$. The $\mathrm{IC}_{50}$ value of Pep-NPSPION/PTX and NP-SPION/PTX is 10.2 and $19.4 \mu \mathrm{g} / \mathrm{mL}$, respectively. 


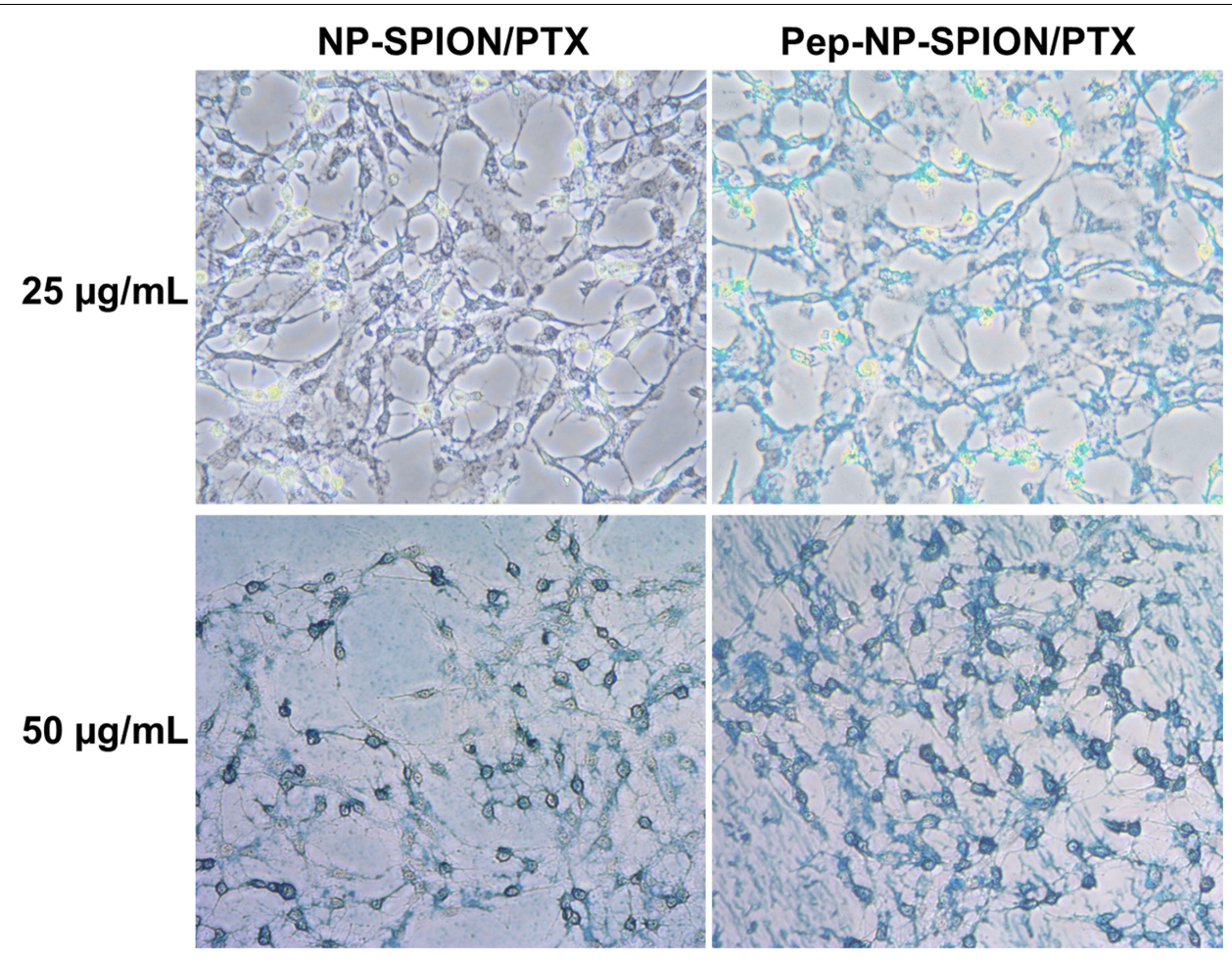

FIGURE 5 | Prussian blue staining images of C6 cells after $1 \mathrm{~h}$ incubation with NP-SPION/PTX and Pep-NP-SPION/PTX with Fe concentration at 25 and $50 \mu$ g/mL. Original magnification: $200 \times$.

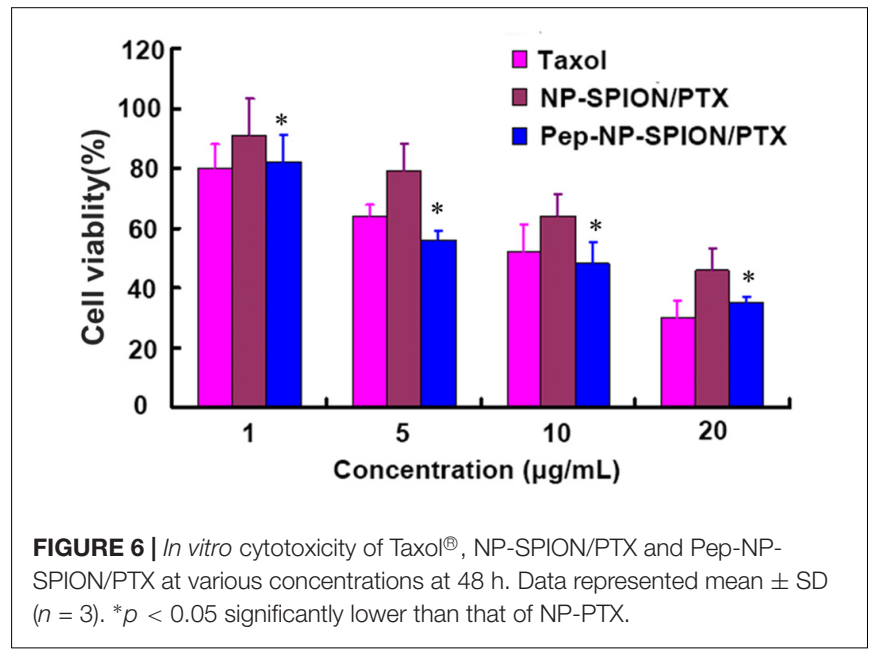

\section{Biodistribution of Pep-NP-SPION/PTX in Tumor Tissue}

The in vivo tumor targeting capability of magnetic nanoparticles was studied qualitatively by Prussian blue staining. Iron oxide was indicated as blue and cell nucleus as red. As shown in Figure 7, in the presence of an external magnetic field, both NP-SPION/PTX and Pep-NP-SPION/PTX exhibited much higher tumor distribution than those without magnetic field intervention, which indicated that the constructed nanoparticles had an attractive magnetic targeting character for improving tumor distribution. Moreover, compared with the NP-SPION/PTX group, Pep-NP-SPION/PTX showed an obvious higher distribution no matter whether an external magnetic field was added, which revealed that the modification of Pep-1 peptide could accelerate the accumulation of nanoparticles into the tumor section via IL-13R $\alpha 2$ mediated endocytosis. Altogether, based on the active targeting and magnetic targeting, Pep-NP-SPION/PTX can be used as a potential dual targeting nanocarrier for the treatment of tumors.

\section{In vivo Anti-tumor Efficacy}

In this study, mice bearing subcutaneous tumor xenograft were used to evaluate the in vivo anti-tumor efficacy of Pep-NP-SPION/PTX. The mice were intravenously injected with Taxol, NP-SPION/PTX and Pep-NP-SPION/PTX (PTX dose: $4.5 \mathrm{mg} / \mathrm{kg}$, Fe dose: $15.6 \mathrm{mg} / \mathrm{kg}$ ) every other day for four consecutive administrations with tumor sizes recorded every 3 days, respectively. As shown in Figure 8, the tumor size of the saline group was obviously all larger than that of the PTX formulations. In the initial days, there was no significant difference in tumor size among these groups. At the experimental terminal, the tumor size of all PTX formulations followed the order: Pep-NP-SPION/PTX with magnetic field $<$ Pep-NP-SPION/PTX $<$ NP-SPION/PTX with magnetic field $<$ NP-SPION/PTX $<$ Taxol $<$ Saline. These results showed that the modification of Pep-1 peptide could improve the anti-tumor efficacy of nanoparticles through IL-13R $\alpha 2$-mediated 


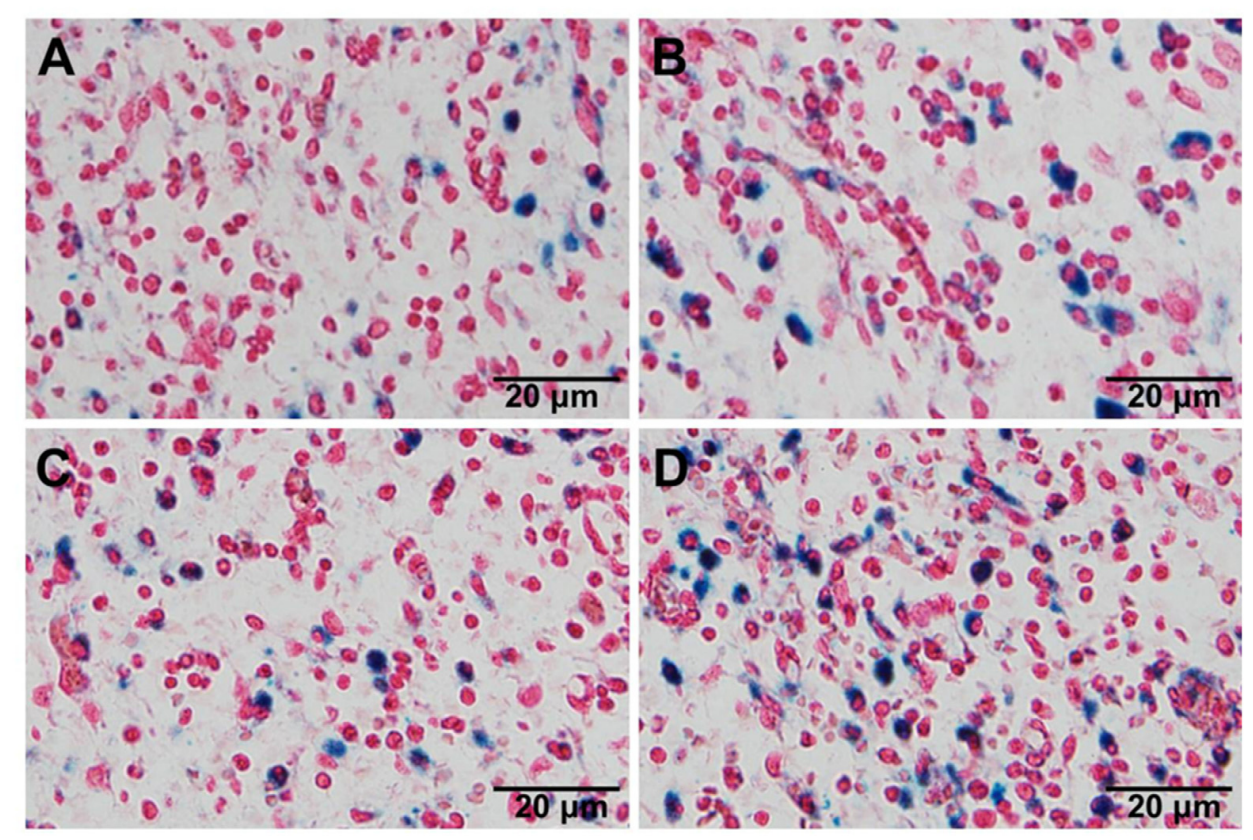

FIGURE 7 | The distribution of nanoparticles in tumor section visualized by Prussian blue staining. Histological sections were extracted at $2 \mathrm{~h}$ after intravenous injection of NP-SPION/PTX (A), NP-SPION/PTX with magnetic field (B), Pep-NP-SPION/PTX (C), and Pep- NP-SPION/PTX with external magnetic field (D).

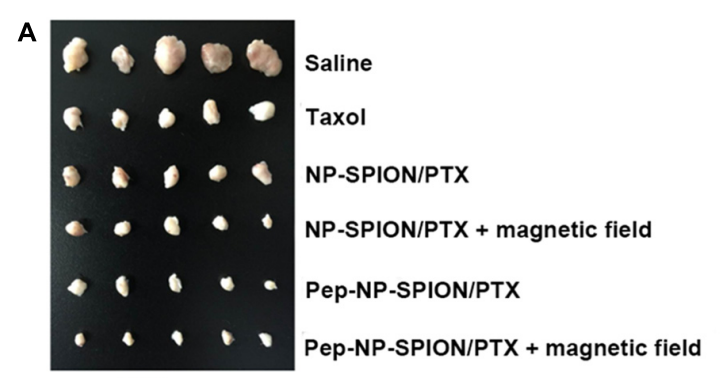

B

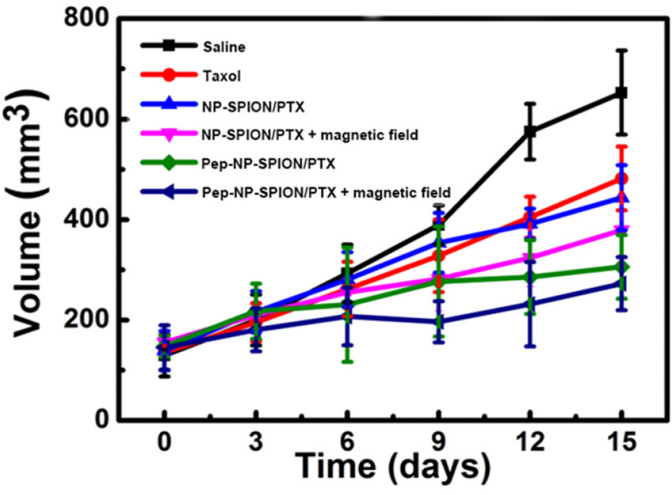

FIGURE 8 | Anti-tumor efficacy of Taxol${ }^{\circledR}$, NP-SPION/PTX and

Pep-NP-SPION/PTX on tumor xenograft mice. Tumor xenografts alignment of each group taken out from the sacrificed mice at the end point (A) and tumor growth volume vs. time (B). $(n=5)$.

endocytosis, consistent with our previous study. Moreover, the encapsulation of SPION endowed nanoparticles with the magnetic targeting property and enhanced the anti-tumor efficacy with the magnetic field. Together, Pep-NP-SPION/PTX constructed in this study could offer a potential magnetic targeting and receptor mediated targeting to enhance the antitumor efficacy for tumor treatment.

\section{CONCLUSION}

In this study, we successfully constructed PTX and SPION coloaded polymer nanoparticles with Pep-1 peptide modification as a dual targeted nanocarrier for tumor treatment. The cellular uptake of Pep-NP-SPION/PTX showed a concentrationdependent manner and significantly enhanced than that of unmodified NP-SPION/PTX. Pep-NP-SPION/PTX exhibited cytotoxicity comparable to Taxol at the PTX concentrations ranging from 1 to $20 \mu \mathrm{g} / \mathrm{mL}$ in vitro cell experiments. Furthermore, Pep-NP-SPION/PTX showed a satisfactory tumor accumulation and the magnetic field significantly enhanced the bio-distribution of nanoparticles in the tumor section. More importantly, the in vivo anti-tumor efficacy showed that Pep-NP-SPION/PTX exhibited desirable anti-tumor efficacy and the magnetic field could also enhance the anti-tumor efficacy. Altogether, these results indicate that the modification of Pep-1 peptide could enhance the active targeting property through receptor-mediated endocytosis and the encapsulation of SPION which obviously improves the physical targeting property of nanoparticles under an external magnetic field. The physical magnetic targeting and IL-13R $\alpha 2$ mediated active targeting characteristics could synergistically increase the targeted efficiency for a tumor. Therefore, Pep-NP-SPION/PTX could serve as a potential dual targeting nanocarrier for tumor therapy. 


\section{AUTHOR CONTRIBUTIONS}

HX designed the experiments. BW and WW performed the experiments. BW and HX wrote the main manuscript. HL and ZW prepared the figures and tables. All authors reviewed the manuscript.

\section{FUNDING}

This work was supported by the grants from the National Natural Science Foundation of China (31671018), the Natural Science

\section{REFERENCES}

Balyasnikova, I. V., Wainwright, D. A., Solomaha, E., Lee, G., Han, Y., Thaci, B., et al. (2012). Characterization and immunotherapeutic implications for a novel antibody targeting interleukin (IL)-13 receptor $\alpha 2$. J. Biol. Chem. 287, 3021530227. doi: 10.1074/jbc.M112.370015

Daines, M. O., Tabata, Y., Walker, B. A., Chen, W., Warrier, M. R., Basu, S., et al. (2006). Level of expression of IL-13R $\alpha 2$ impacts receptor distribution and IL-13 signaling. J. Immunol. 176, 7495-7501. doi: 10.4049/jimmunol.176.12.7495

Du, W. W., Fan, Y. C., He, B., Zheng, N., Yuan, L., Dai, W. B., et al. (2015). Bionano interactions of MCF-7 breast tumor cells with a transferrin receptor targeted nanoparticle. Mol. Pharmacol. 12, 1467-1476. doi: 10.1021/mp500796d

Hachani, R., Lowdell, M., Birchall, M., Hervault, A., Mertz, D., Begin-Colin, S., et al. (2016). Correction: polyol synthesis, functionalisation, and biocompatibility studies of superparamagnetic iron oxide nanoparticles as potential MRI contrast agents. Nanoscale 8, 3278-3287. doi: 10.1039/c5nr03867g

Huang, W. C., Chen, S. H., Chiang, W. H., Huang, C. W., Lo, C. L., Chern, C. S., et al. (2016). Tumor microenvironment-responsive nanoparticle delivery of chemotherapy for enhanced selective cellular uptake and transportation within tumor. Biomacromolecules 17, 3883-3892. doi: 10.1021/acs.biomac.6b00956

Kawakami, K., Taguchi, J., Murata, T., and Puri, R. K. (2001). The interleukin13 receptor $\alpha 2$ chain: an essential component for binding and internalization but not for interleukin-13-induced signal transduction through the STAT6 pathway. Blood 97, 2673-2679. doi: 10.1182/blood.V97.9.2673

Li, L., Gao, F. M., Jiang, W. W., Wu, X. L., Cai, Y. Y., Tang, J. T., et al. (2016). Folic acid-conjugated superparamagnetic iron oxide nanoparticles for tumortargeting MR imaging. Drug Deliv. 23, 1726-1733. doi: 10.3109/10717544.2015. 1006404

Mintz, A., Gibo, D. M., Slagle-Webb, B., Christensen, N. D., and Debinski, W. (2002). IL-13Ralpha2 is a glioma-restricted receptor for interleukin-13. Neoplasia 4, 388-399. doi: 10.1038/sj.neo.7900234

Mirsadeghi, S., Shanehsazzadeh, S., Atyabi, F., and Dinarvand, R. (2016). Effect of PEGylated superparamagnetic iron oxide nanoparticles (SPIONs) under magnetic field on amyloid beta fibrillation process. Mater. Sci. Eng. C Mater. Biol. Appl. 59, 390-397. doi: 10.1016/j.msec.2015.10.026

Muralidharan, R., Babu, A., Amreddy, N., Srivastava, A., Chen, A., Zhao, Y. D., et al. (2017). Tumor-targeted nanoparticle delivery of HuR siRNA inhibits lung tumor growth In Vitro and In Vivo by disrupting the oncogenic activity of the RNA-binding protein HuR. Mol. Cancer Ther. 16, 1470-1486. doi: 10.1158/ 1535-7163.MCT-17-0134

Nagesh, P. K. B., Johnson, N. R., Boya, V. K. N., Chowdhury, P., Othman, S. F., Khalilzad-Sharghi, V., et al. (2016). PSMA targeted docetaxel-loaded superparamagnetic iron oxide nanoparticles for prostate cancer. Colloids Surf. B Biointerfaces 144, 8-20. doi: 10.1016/j.colsurfb.2016.03.071

Pandya, H., Gibo, D. M., Garg, S., Kridel, S., and Debinski, W. (2012). An interleukin 13 receptor a2-specific peptide homes to human Glioblastoma multiforme xenografts. Neuro Oncol. 14, 6-18. doi: 10.1093/neuonc/ nor141

Rabiej, V. K., Pflanzner, T., Wagner, T., Goetze, K., Storck, S. E., Eble, J. A., et al. (2016). Low density lipoprotein receptor-related protein 1 mediated endocytosis of $\beta 1$-integrin influences cell adhesion and cell migration. Exp. Cell Res. 340, 102-115. doi: 10.1016/j.yexcr.2015.11.020
Foundation of Jiangsu Province-Excellent Young Scientist Fund (BK20160096), the 2016 Qing Lan Program of Jiangsu Province, and the 2017 Six Talent Peaks Project of Jiangsu Province.

\section{ACKNOWLEDGMENTS}

The authors acknowledge the support from the School of Pharmacy, Fudan University and the Open Project Program of Key Lab of Smart Drug Delivery (Fudan University), Ministry of Education, China (SDD2012-4).

Roberts, C. M., Shahin, S. A., Wen, W., Finlay, J. B., Dong, J., Wang, R., et al. (2017). Nanoparticle delivery of siRNA against TWIST to reduce drug resistance and tumor growth in ovarian cancer models. Nanomedicine 13, 965-976. doi: 10. 1016/j.nano.2016.11.010

Schleich, N., Sibret, P., Danhier, P., Ucakar, B., Laurent, S., Muller, R. N., et al. (2013). Dual anticancer drug/superparamagnetic iron oxide-loaded PLGAbased nanoparticles for cancer therapy and magnetic resonance imaging. Int. J. Pharm. 447, 94-101. doi: 10.1016/j.ijpharm.2013.02.042

Shen, Z. Q., Ye, H. L., Kröger, M., and Li, Y. (2018). Aggregation of polyethylene glycol polymers suppresses receptor-mediated endocytosis of PEgylated liposomes. Nanoscale 10, 4545-4560. doi: 10.1039/c7nr09011k

Silva, A. H., Lima, E. Jr., Mansilla, M. V., Zysler, R. D., Troiani, H., Pisciotti, M. L. M., et al. (2016). Superparamagnetic iron-oxide nanoparticles mPEG350and mPEG2000-coated: cell uptake and biocompatibility evaluation. Nanomedicine 12, 909-919. doi: 10.1016/j.nano.2015.12.371

Song, H. J., Zhang, J., Wang, W. W., Huang, P. S., Zhang, Y. M., Liu, J. F., et al. (2015). Acid-responsive PEGylated doxorubicin prodrug nanoparticles for neuropilin-1 receptor-mediated targeted drug delivery. Colloids Surf. B Biointerfaces 136, 365-374. doi: 10.1016/j.colsurfb.2015.09.030

Wang, B. Y., Lv, L. Y., Wang, Z. Y., Zhao, Y., Wu, L., Fang, X. L., et al. (2014). Nanoparticles functionalized with Pep-1 as potential glioma targeting delivery system via interleukin 13 receptor $\alpha 2$-mediated endocytosis. Biomaterials 35 , 5897-5907. doi: 10.1016/j.biomaterials.2014.03.068

Wang, X., Li, J., Wang, Y. X., Koenig, L., Gjyrezi, A., Giannakakou, P., et al. (2011). A folate receptor-targeting nanoparticle minimizes drug resistance in a human cancer model. ACS Nano 5, 6184-6194. doi: 10.1021/nn200739q

Wilhelm, S., Tavares, A. J., Dai, Q., Ohta, S., Audet, J., Dvorak, H. F., et al. (2016). Analysis of nanoparticle delivery to tumours. Nat. Rev. Mater. 1:16014. doi: 10.1038/natrevmats.2016.14

Wong, M. H., Chan, D. L., Lee, A., Li, B. T., Lumba, S., Clarke, S. J., et al. (2016). Systematic review and meta-analysis on the role of chemotherapy in advanced and Metastatic Neuroendocrine Tumor (NET). PLoS One 11:e0158140. doi: 10.1371/journal.pone.0158140

Xu, Q., Liu, Y. X., Su, S. S., Li, W., Chen, C. Y., and Wu, Y. (2012). Antitumor activity of paclitaxel through dual-targeting carrier of cyclic RGD and transferrin conjugated hyperbranched copolymer nanoparticles. Biomaterials 33, 1627-1639. doi: 10.1016/j.biomaterials.2011.11.012

Zhao, R. B., Visentin, M., and Goldman, I. D. (2015). Determinants of the activities of antifolates delivered into cells by folate-receptor-mediated endocytosis. Cancer Chemother. Pharmacol. 75, 1163-1173. doi: 10.1007/s00280-015-2733-8

Conflict of Interest Statement: The authors declare that the research was conducted in the absence of any commercial or financial relationships that could be construed as a potential conflict of interest.

Copyright (c) 2019 Wang, Wu, Lu, Wang and Xin. This is an open-access article distributed under the terms of the Creative Commons Attribution License (CC BY). The use, distribution or reproduction in other forums is permitted, provided the original author(s) and the copyright owner(s) are credited and that the original publication in this journal is cited, in accordance with accepted academic practice. No use, distribution or reproduction is permitted which does not comply with these terms. 